\title{
Performance Comparison of SVC with POD and Synchronous Generator Excitation System to Investigate Oscillation Damping Control on the GB Transmission System
}

\author{
Ronak Rabbani \\ Brunel University \\ Ronak.Rabbani@brunel.ac.uk
}

\author{
Ahmed F. Zobaa \\ Brunel University \\ Ahmed.Zobaa@brunel.ac.uk
}

\author{
Gareth A. Taylor \\ Brunel University \\ Gareth.Taylor@brunel.ac.uk
}

\begin{abstract}
Energy and environmental issues are two of the greatest challenges facing the world today. In response to energy needs and environmental concerns, renewable energy technologies are now considered the future technologies of choice. Renewable energy is produced from natural sources that are clean and free; however, it is widely accepted that renewable energy is not a solution that is without challenges. An example of this can be seen in the UK where there is much interest amongst generation developers in the construction of new large-scale onshore and offshore wind farms, especially in Scotland.

As a part of an analysis of HVDC operating in parallel with AC transmission lines, this paper investigates PSS and SVC oscillation damping control schemes. The simple models presented in this paper represent the HVDC link and SVC as elements within the power system which provide an acceptable interaction with other system elements.

In this study, in order to verify the performance of embedded HVDC links in parallel with the AC system and in combination with SVC, and the effect of the IEEE standard excitation model systems, an appropriate simulation for the system is performed using the PSCAD/EMTDC program.
\end{abstract}

Index Terms--SVC POD Controller, PSS1A Type PSS, IEEE standard excitation model, PSCAD/EMTDC

\section{INTRODUCTION}

The UK government has made wind energy a key tool in its commitment to reduce anthropomorphic greenhouse gas emission levels. In particular, the number of large offshore and onshore wind farms in the UK is expected to increase considerably [1, 2] and there will be key operational challenges for the Great Britain (GB) network with increased wind penetration in northern Scotland [3]. Therefore, it is planned to reinforce the GB electrical power transmission system between 2013 and 2021 through the use of many more HVDC links operating in parallel with existing AC transmission routes, and also controllable reactive power sources such as SVC and TCSCs.

High Voltage DC (HVDC) transmission and conventional static VAR compensators (SVC) are established technologies; however, HVDC has recently become an important way of linking long distance interconnections. Additionally, controllable reactive power sources, such as SVCs, because of their ability to control bus voltage and improve the maximum power transfer limits available on the transmission line, are gaining increasing popularity in the field of power transmission and distribution.

The main power oscillation damping system which is used in conventional power grids is Power System Stabilization (PSS), but it is not appropriate for all problems. Consequently, to increase the reliability of the power system, other FACTs devices are required [4]. SVCs are examples of shunt compensators, which are used to maintain voltage magnitude. The PI controller parameter of a SVC is of fundamental importance in order to damp the power system oscillation of a synchronous generator [5].

In contrast, the excitation system is required to make an impact on the oscillations in the connected network. Speed of action and maximisation of the synchronous torque of the generating unit are characteristics of these excitation systems. After a transient fault has occurred characteristics such as these lead to a rotor movement that becomes stable, and returns to its steady state position. In addition, this kind of excitation system during a fault, can contribute to a high terminal voltage that leads to a high current. It is appropriate to maintain a high current in order to improve the tripping ability of protective relays [6].

This paper proposes a range of dynamic stability analysis techniques using a simple model with regard to disturbance in the transmission system. This simple model represents the HVDC and SVC as an element in the power system that acceptably interacts with other system elements. This system is considered in order to test the control and this simple model which incorporates a control system, will be useful in the future for implementation in a reduced model of the GB transmission system. The impact of embedded HVDC links in parallel with the existing $\mathrm{AC}$ system and in combination with SVCs and an IEEE standard excitation system is investigated with regard to active power flow. The most important point is to ensure the stability of the system when a credible contingency occurs. 
In this paper, the test system modelling is described in section II and the SVC control scheme is presented in section III. Section IV presents the results of the time domain simulation on the PSCAD/EMTDC program, and finally, the conclusions and future work are proposed in section $\mathrm{V}$.

\section{PSCAD/EMTDC POWER SYSTEM MODEL}

The modelled power system is basically one generator and an infinite bus system. This model is included two areas which are connected via one AC transmission line in parallel with a HVDC line. In addition there is one SVC at the generators bus.

\section{A. Generator Model}

The windmills are represented by synchronous machines in the PSCAD/EMTDC library and all the parameters are defined as in Table I.

TABLE I

GENERATOR DATA

\begin{tabular}{|l|l|}
\hline Armature Time Constant [Ta] & 0.278 [sec] \\
\hline Potier Reactance [Xp] & 0.15 [p.u.] \\
\hline Unsaturated Reactance [Xd] & 1.94 [p.u.] \\
\hline Unsaturated Transient Reactance [Xd'] & 0.2259 [p.u.] \\
\hline Unsat. Transient Time (Open) [Tdo'] & 10.4 [sec] \\
\hline Unsat. Sub-Trans. Reactance [Xd''] & 0.1723 [p.u.] \\
\hline Unsat. Sub-Trans. Time (Open) [Tdo''] & 0.03 [sec] \\
\hline Unsaturated Reactance [Xq] & 1.92 [p.u.] \\
\hline Unsaturated Reactance [Xq'] & 0.402 [p.u.] \\
\hline Unsat. Trans. Time (Open) [Tqo'] & 0.83 [sec] \\
\hline Unsat. Sub-Trans. Reactance [Xq''] & 0.1723 [p.u.] \\
\hline Unsat. Sub-Trans. Time (Open) [Tqo'] & 0.055 [sec] \\
\hline Air Gap Factor & 1 \\
\hline
\end{tabular}

\section{B. HVDC modelling}

The HVDC model, based on the PSCAD/EMTDC model, includes two 6-pulse converters, AC filters and DC filters. Each terminal of the HVDC link includes two 6-pulse converters connected in series in order to produce a high voltage on the DC transmission line $(400 \mathrm{kV})$. Each converter is connected to the AC system through a transformer. One transformer has a star connection, while the other has a delta connection at the primary side.

The DC current is smoothed by two inductors on two sides of the DC link. Low and high frequency filters are used to reduce the harmonics in the $\mathrm{AC}$ voltage and to provide reactive power for the converters. Apart from the filters, the reactive power compensation is supplied by the capacitor banks on each side of the HVDC interconnection.

\section{SVC modelling}

The SVC model in the PSCAD library is based on a 12pulse thyristor-switched capacitor (TSC), and a thyristorcontrolled reactor (TCR). Its transformer consists of a star connected primary winding and dual secondary windings; one star and one delta connected. User required input control signals to the SVC model include the thyristor firing order, capacitor switching signal (to add or remove a capacitor stage), and the 'block/de-block' signal (enable). SVC simulation output variables can be viewed and plotted, including the number of capacitor stages currently in use and the thyristor firing order [7].

\section{Exciter Modelling ( Static Excitation System)}

To control the performance of the synchronous machine, the main part which has to be controlled is the DC rotor current, which is achieved through an automatic voltage regulator (AVR). The gate opening of the thyristors in the controlled rectifier is controlled by this AVR. The typical excitation system block diagram is illustrated in Fig.1. It can be seen that in order to calculate a voltage error signal the generator voltage is measured and compared with a reference voltage. As a result, the correct AC generator terminal voltage will be achieved by regulating this signal to give the wanted DC output voltage of the exciter (Ef) [6].

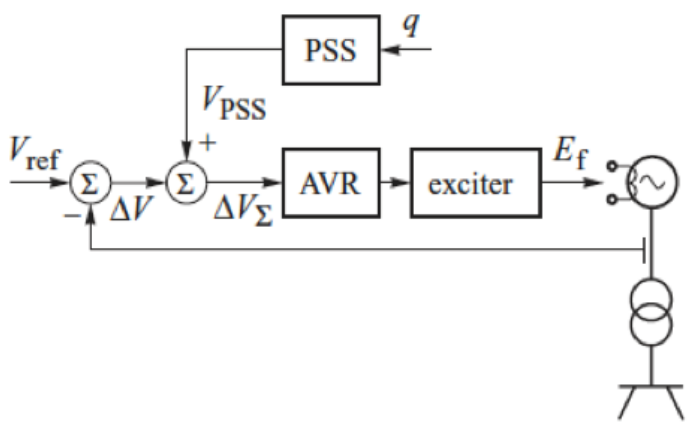

Fig.1. Block diagram of the excitation system of one generator connected to the grid [6].

For this study different types of IEEE excitation modelling systems which exist in the PSCAD/EMTDC library are used. In these excitation systems voltage and current in compounded systems are changed to an appropriate level. In either a controlled or non-controlled system, the essential direct current for the generator field is provided by the rectifiers. For the some excitation systems which have very high levels of exciter ceiling voltage, additional field current limiter circuits may be used to protect the exciter and the generator rotor. These current limiter circuits will be used for both instantaneous and time delayed elements, but only the instantaneous limits are included in this model and only for the ST1A and ST6B models [8].

\section{- Type ST1A Excitation System Model:}

This type of excitation system is proposed to represent systems in which the excitation power is supplied through a transformer from the generator terminals and regulated by a controlled rectifier. For this system the maximum exciter voltage which is available from such systems is dependent upon the generator terminal voltage. In this type of system, the exciter stabilization may not be required because the characteristic exciter time constants are very small, but to reduce the transient gain of these systems for other reasons 
might be necessary [8].

\section{- Type ST2A Excitation System Model:}

For this type of excitation system the power source is comprised of both the current and voltage sources. This is needed to form a model of the exciter power source operating a phasor combination of terminal voltage and terminal current [8].

\section{- Type ST3A Excitation System Model:}

In this excitation system for linearising the exciter control characteristic, a field voltage control loop is developed. Because of this operation the output is independent of supply source variations until the supply limitations are reached. In this excitation model full thyristor complements or hybrid bridges in either series or shunt configurations are utilised, which is a variation of controlled-rectifier designs. The potential source, either fed from the machine terminals or from internal windings, may be used as the power source. However, both the machine potential and current can be used for designing compound power sources utilising some design. The time constants TB and TC are the series lag-lead element in the voltage regulator. These parameters provide the excitation system stabiliser for this type of excitation [8].

\section{- Type ST5B Excitation System Model:}

The variation of the type ST1A model with alternative overexcitation and under-excitation inputs and additional limits is presented as a type ST5B excitation system model. The stabiliser models Type PSS2B, PSS3B or PSS4B can be used with these models [8].

\section{- Type ST6B Excitation System Model:}

The Type ST6B excitation system consists of a PI voltage regulator with an inner loop field voltage regulator and precontrol, and this proportional control is implemented by the field voltage regulator. The pre-control and the delay in the feedback circuit result in increasing the dynamic response of this model. This type of excitation includes the ceiling current IFD limitation. The power for the rectifier is supplied from the generator terminals or from an independent source. Inputs are provided for external models of the over-excitation limiter (VOEL), under-excitation limiter (VUEL), and PSS (VS) [8].

\section{E. Power System Stabiliser Modelling}

In this simple model, to improve damping of power system oscillations through the excitation control, PSS was used.

The frequency can be used as an input parameter for PSS. Generally it will be terminal frequency, but it can be a frequency behind a simulated machine reactance in some cases. Also for many studies equivalent to shaft speed may be used.

The type of input signal for the stabiliser determines the stabiliser model. For different input signals, parameters for stabilisers may look very different while running similar damping characteristics [8].

\section{- Type PSS1A Power System Stabiliser:}

The general form of a PSS with a single input is shown in Fig.2. $\mathrm{V}_{\mathrm{SI}}$ or the input signal for this model can be speed, frequency or power.

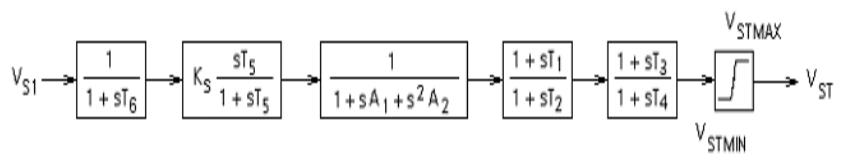

Fig.2. Block diagram for PSS1A- Single input power system stabiliser [9].

T6 in the first block is used to represent a transducer time constant. In the second block the stabiliser gain is defined by the term $\mathrm{K}_{\mathrm{S}}$ and the signal washout is set by the time constant T5. A1 and A2 in the third block, which are used in some stabilisers, admit some of the low-frequency effects of highfrequency torsional filters which should be accounted for. For others studies this block can be used to set the gain and phase characteristics of the stabiliser if required. The next two blocks are used for two stages of lead-lag compensation as set by the constants $\mathrm{T} 1$ to $\mathrm{T} 4$.

The stabiliser output or $\mathrm{V}_{\mathrm{ST}}$, is an input to the supplementary discontinuous control models and where the discontinuous control models are not used, then $\mathrm{V}_{\mathrm{ST}}$ will be VS [8].

\section{- Type DEC1A Discontinuous Excitation Control:}

Fig.3 shows the Type DEC1A discontinuous excitation control model. This control scheme is used specifically for cases where the local and inter area oscillations may be presented in the transient stability study.

It can be seen in this block diagram that, when a fault occurs in the system, by using the voltage regulator and stabiliser the level of generator excitation will be increased to higher than that demanded following the system fault. In this control system a proportional signal is added to the rotor angle change to the terminal voltage and power system stabilising signals. This angle signal will be the source of instability if it is used continuously. As a result, this signal is used for only approximately $2 \mathrm{~s}$ during the transient period $[8]$. 


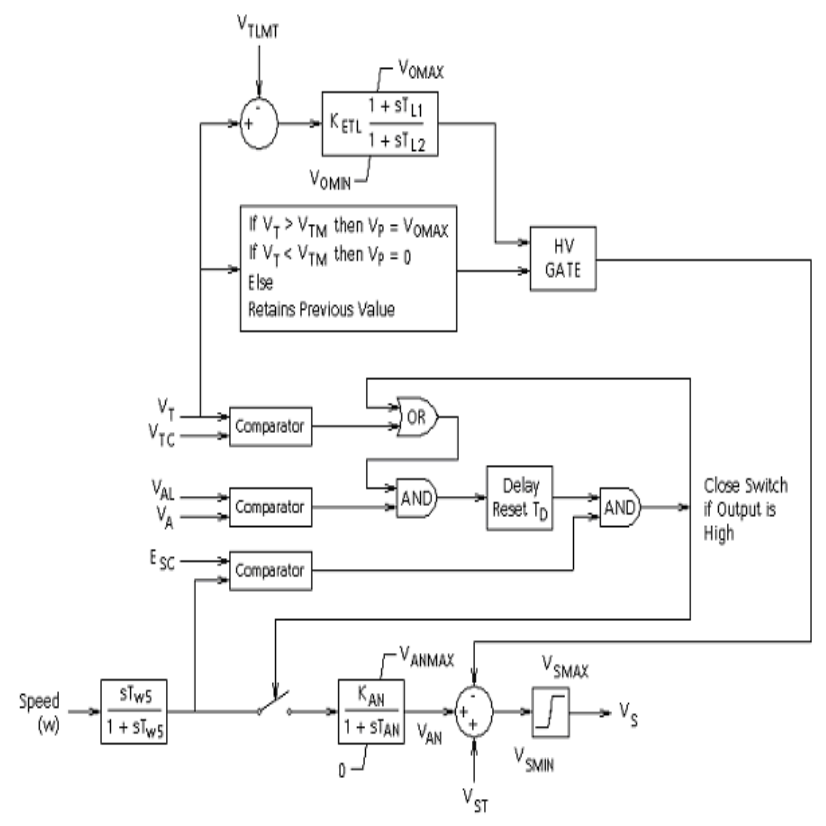

Fig.3. Block diagram for DEC1- Transient Excitation Booster with Dual Action Terminal Voltage Limiter [9].

Achieving the maximum of the rotor angle swing is the main objective of this control scheme, which will be reached by keeping the terminal voltage high. Extreme terminal voltage is avoided through use of a terminal voltage limiter circuit.

Furthermore, the effect of this discontinuous control is to increase the system voltage level and therefore load power, thereby contributing to unit checking [8].

\section{SVC POD CONTROL SYSTEM}

The power oscillation damping (POD) controller for SVC is provided in order to improve the damping of the power system oscillation. The damping controller may be considered as including two cascade-connected blocks as shown in Fig. 4.

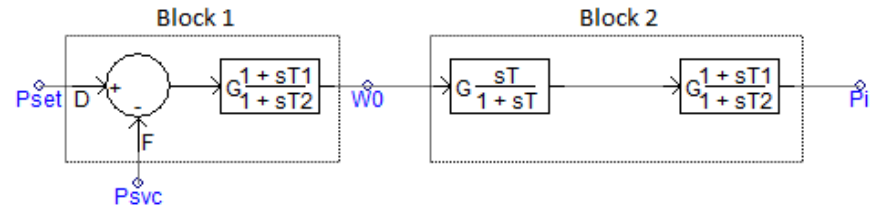

Fig. 4. Structure of Power Oscillation damping Controller for SVC

It can be observed that block 1 is provided in order to derive the speed deviation signal from the electric power. In this block the total electrical power that is measured at the SVC location is compared with the set point (mechanical power). To obtain a speed deviation signal, the error is integrated and multiplied by $1 / 2 \mathrm{H}$.

As the speed deviation signal in general may not be available at the SVC location, the speed deviation signal derived uses instead the speed deviation signal [10].
At block 2 a lead-lag compensator is present. In order to improve the damping of the power system oscillation, an electric torque in phase with the speed deviation is also present.

The electrical power deviation is the result of balancing the phase shifting between control signals. The parameters of the lead-lag compensator are chosen so as to balance for this phase shift.

Therefore, an additional electrical power output is obtained in phase with the speed deviation. The required damping ratio of the electromechanical mode is defined by setting the gain of the damping controller.

The Pi or output of the damping controller for the SVC modifies the reference setting of the power flow controller [10].

\section{Simulation RESUlts}

In order to verify the performance of the embedded HVDC links in parallel with the AC system and in combination the SVC, and the effect of the different static excitation systems and the proposed POD controller SVC, an appropriate simulation for the simplified system as described in section II was performed on a PSCAD/EMTDC. Among different short circuit faults, a three-phase short circuit fault was the most severe [11], so in this paper a three-phase fault was modelled in the rectifier terminal of the HVDC interconnection at $12.8 \mathrm{~s}$ which was cleared in $0.25 \mathrm{~s}$. The assessments of the controller used and the IEEE standard excitation models are analysed through the observations of the active power flow of the PCC transmission line. Fig. 5 shows the resultant waveform for the system without PSS and POD control systems for the SVC.

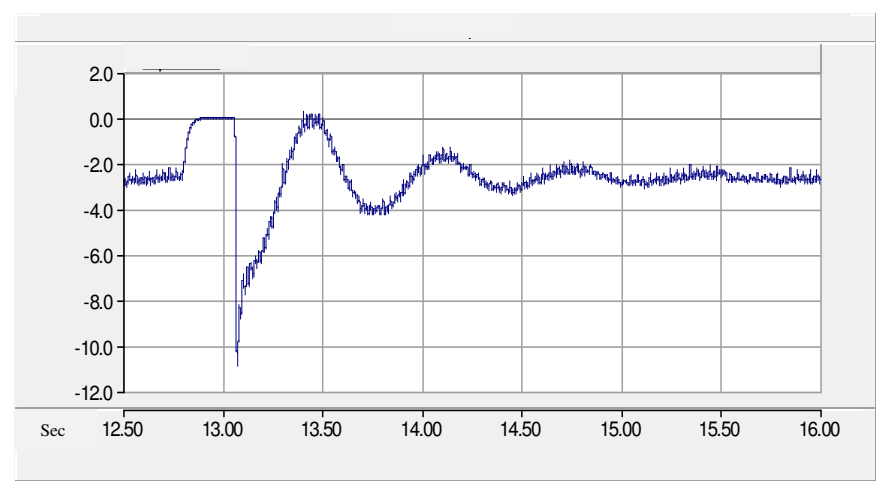

Fig. 5. Active power flow at PCC point without PSS and POD control systems for the SVC.

\section{A. Type STIA Excitation System:}

As a first case, Fig. 6 shows the waveform for the system with SVC power oscillation damping control and ST1A excitation model. For this excitation the data is defined as flow [8]:

$\begin{array}{lll}K A=210.0 & T B 1=0 & K F=0 \\ T A=0 & V R M A X=6.43 & T C=1.0 \\ \text { VRMIN }=-6.0 & \text { KLR }=4.54 & T B=1.0 \\ K C=0.038 & I L R=4.4 & T C 1=0\end{array}$


The simulations demonstrated are for active power flow at the PCC point. It can be seen that the time required for the response curve to reach and remain within a range of a certain percentage $(2 \%)$ of the final value, the setting time or $\mathrm{T}_{\mathrm{S}}$ is $15 \mathrm{~s}$.

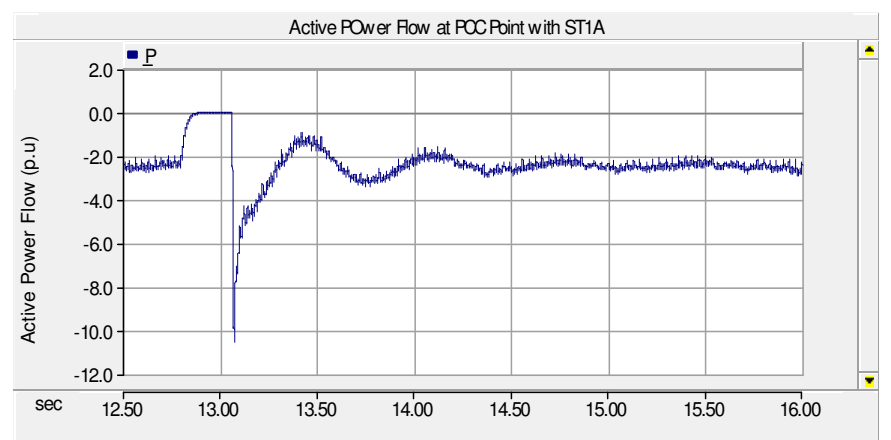

Fig. 6. Active power flow at PCC point with ST1A excitation model system.

\section{B. Type ST2A Excitation System:}

For this step ST2A was used as an excitation model for the synchronous generator. Fig. 7 shows the results for an active power flow at the PCC point. The series lag-lead element in the voltage regulator provides the excitation system stabiliser for these systems. This element in the voltage regulator is represented by the time constants TB and TC. The inner loop has a wide bandwidth compared with the upper limit of $3 \mathrm{~Hz}$ for this excitation system model. For this excitation the data is defined as flow [8]:

$$
\begin{array}{lll}
K A=190 & T B 1=0 & T A=0 \\
V R M A X=7.8 & K F=0 & T C=1.0 \\
\text { VRMIN }=-6.7 & T B=10.0 & \text { KC }=0.08 \\
\text { KLR }=0 & T C 1=0 & \text { VIMAX }=999
\end{array}
$$

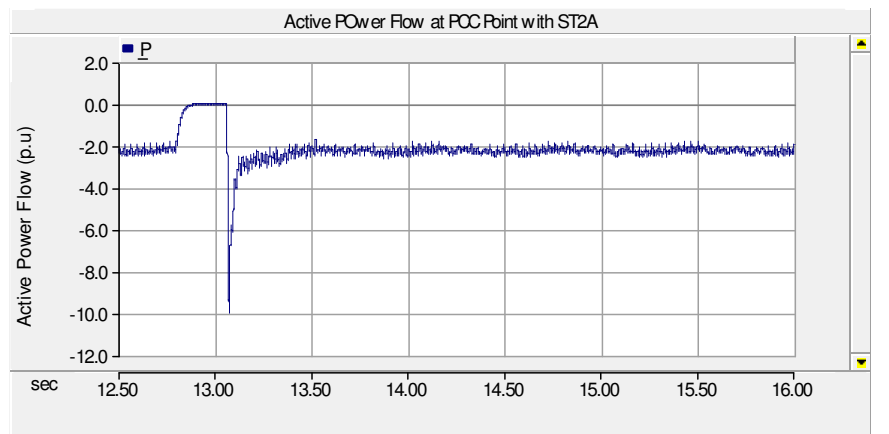

Fig.7. Active power flow at PCC point with ST2A excitation model system.

It can be seen for this case that the time required for the response curve to reach and remain within a certain percentage range $(2 \%)$ of the final value, the $T_{S}$ is $13.5 \mathrm{~s}$, which is a significant improvement when compared to the other cases. The steady state error and overshoot is also satisfactorily reduced.
C. Type ST3A Excitation System:

The results obtained for Type ST3A is showed in Fig. 8 and the data for ST3A was also defined as flow [8]:

$\begin{array}{lll}T A=0 & V I M I N=-0.2 & K G=1.0 \\ T R=0 & V M M A X=1.0 & K M=7.93 \\ T M=0.4 & V M M I N=0 & K A=200 \\ T B=10.0 & V R M A X=10.0 & K P=6.15 \\ T C=1.0 & V R M I N=-10.0 & \left(P=0^{\circ}\right. \\ X_{L}=0.081 & V G M A X=5.8 & 4 K I=0 \\ V I M A X=0.2 & \text { EFD MAX }=6.9 & K C=0.20\end{array}$

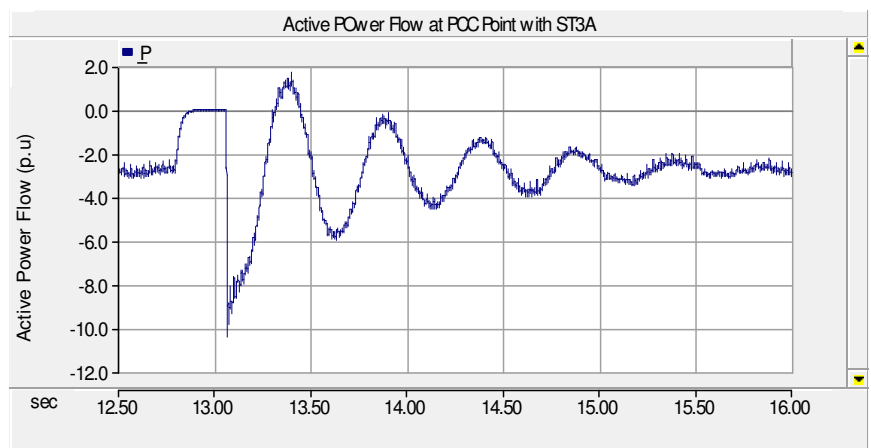

Fig. 8. Active power flow at PCC point with ST3A excitation model system.

It can be observed that for type ST3A the excitation model system overshoot is increased and the $T_{S}$ is $16.5 \mathrm{~s}$, which is more than the other examples presented in this paper.

\section{Type ST5B Excitation System:}

The IEEE type ST5B excitation model system in block diagram form is shown in Fig. 9. This type is a variation of the Type ST1A model, with alternative over-excitation and under-excitation inputs and additional limits [8].

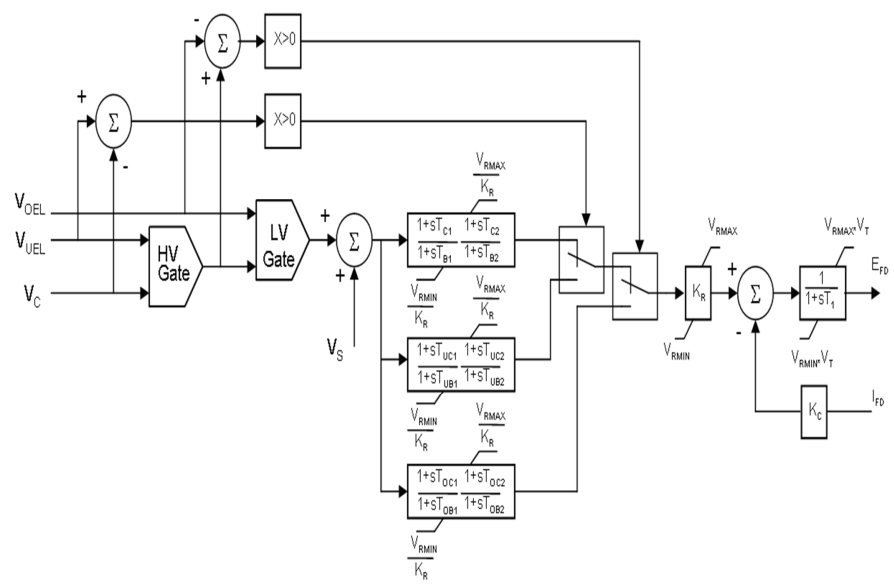

Fig. 9. Type ST5B—Static potential-source excitation system [8]

The data for this model was also defined as flow [8]:

$\begin{array}{lll}\text { TB1 }=6.0 & \text { TC1 }=0.8 & \text { TB2 }=0.01 \\ \text { TC2 }=0.08 & \text { TUB } 1=10 & \text { TUC1 }=2 \\ \text { TUB2 }=0.05 & \text { TUC2 }=0.1 & \text { TOB } 1=2 \\ \text { TOC1 }=0.1 & \text { TOB2 }=0.08 & \text { TOC2 }=0.08 \\ \text { KR }=200.0 & \text { T } 1=0.004 & \text { VRmax }=5.0\end{array}$




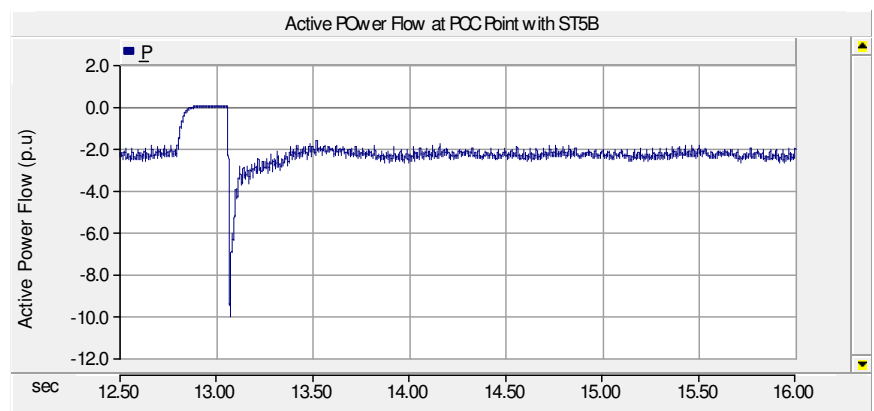

Fig.10. Active power flow at PCC point with ST5B excitation model system.

Fig.10 shows the results obtained and reveals a significant improvement for the overshoot and setting time. Here, the overshoot is the same as that achieved for the Type ST2A excitation model system but the time required for the response curve to reach and remain within a certain percentage range $(2 \%)$ of the final value is $13.7 \mathrm{~s}$.

\section{E. Type ST6B Excitation System:}

Fig. 11 represents the waveform of the damping effect of the Type ST6B excitation system which is present in the PSCAD/EMTDC package. The data for this model was defined as flow [8]:

$\begin{array}{lll}K P A=18.038 & K I A=45.094 s-1 & K F F=1 \\ K M=1 & K G=1 & T G=0.02 s \\ T R=0.012 s & \text { VAMAX }=4.81 & \text { VAMIN }=-3.85 \\ K C I=1.0577 & \text { KLR }=17.33 & I L R=4.164 \\ V R M A X=4.81 & \text { VRMIN }=-3.85 & \end{array}$

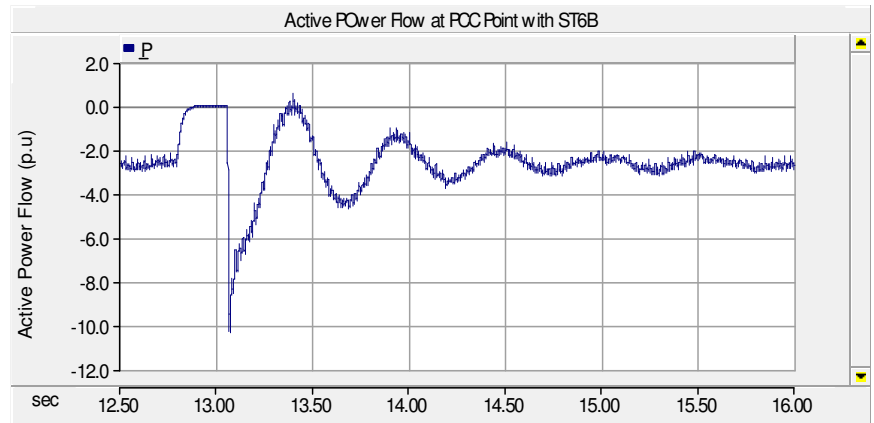

Fig.11. Active power flow at PCC point with ST6B excitation model system.

For this model it can be observed that the $\mathrm{T}_{\mathrm{S}}$ for the active power flow and overshoot are improved compared to the Type ST3A excitation model system, but both are increased when compared to other systems presented in this paper. Here, the time required for the response curve to reach and remain within a certain percentage range $(2 \%)$ of the final value, the $T_{S}$ is $15.7 \mathrm{~s}$

\section{CONCLUSIONS AND FUTURE WORK}

In this paper time domain simulations were used to demonstrate that when AC and DC systems are operated in parallel, then a fault in the AC system leads to an instantaneous power imbalance in the power system, and power shifted through the AC and DC systems impacts greatly on the transient stability. The proposed power system stabiliser (type PSS1A and DEC1A) provides a significant improvement in the power oscillation. In addition, the combination of a PSS and SVC power oscillation damping control system has a significant effect on the setting time and overshoot.

The different excitation system models i.e. Types ST1A, ST2A, ST3A, ST5B and ST6B, have been studied in order to analyse the power oscillation damping and stability of the systems. The simulation test results show that the Types ST2A and ST5B excitation systems have the best effect on power oscillation damping. The overshoot is almost zero for these types and both of them are settled less than 1 second after the occurrence of a fault.

Future work focusing on linking the PSCAD and Matlab programs for the design of advanced power oscillation damping control systems is planned.

\section{REFERENCES}

[1] British Wind Energy Association, www.bwea.com (last accessed Sept 2012)

[2] S.Chondrogiannis, M. Barnes, M. Aten and P. Cartwright, "Modelling and GB grid code compliance studies of offshore wind farms with doubly-fed induction generators," The 3rd IET International Conference on Power Electronics, Machines and Drives, 2006, pp. 22-26, March 2006.

[3] Electricity Networks Strategy Group, Our Electricity Transmission Network: A Vision for 2020, Technical Report URN: 09D/717, July 2009.

[4] Shajari, S. Norouzi, M.R. ; Abedini, A.; Ahi, K., "Coordinate control of TCSC based GA controller with PSS for stability improving in single machine system", Environment and Electrical Engineering (EEEIC), 2011 10th International Conference on 8-11 May 2011

[5] Chandrakar, V.K.; Dhurvey, S.N.; Suke, S.C., "Performance Comparison of SVC with POD and PSS for Damping of Power System Oscillations", Emerging Trends in Engineering and Technology (ICETET), 2010 3rd International Conference on 19-21 Nov. 2010

[6] A. Hammer, "Analysis of IEEE Power System Stabilizer Models", Master of Science in Electric Power Engineering, June 2011

[7] R.R. Peters, D. Muthumunib, T. Bartel, H. Salehfar and M. Manna, "Static VAR compensation of a fixed speed stall control wind turbine during start-up," Electric Power Systems Research, vol. 80 (4), pp. 400-405, April 2010.

[8] IEEE Power Engineering Society, "IEEE Recommended Practice for Excitation System Models for Power System Stability Studies”, April 2006

[9] PSCAD/EMTDC version X4 manual, www.pscad.com/products/pscad (last accessed Sept 2012)

[10] V.K. Chandrakar, S.N. Dhurvey, S. C. Suke "Performance Comparison of SVC with POD and PSS for Damping of Power System Oscillations", Third International Conference on Emerging Trends in Engineering and Technology

[11] I.T. Fernando, W.T. Kwasnicki and A.M. Gole, "Modeling of conventional and advanced static Var compensators in an electromagnetic transient simulation program", unpublished. 